\title{
Correlates of Sedentary Behavior among Bhutanese Adults: Findings from the 2014 Bhutan STEPS Survey Data
}

\author{
Tashi Dendup ${ }^{1, *}$, Yun Zhao' ${ }^{2}$ I Gusti Ngurah Edi Putra', Tandin Dorji ${ }^{3}$, Chador Tenzin ${ }^{4}$ \\ 'School of Health and Society, Faculty of the Arts, Social Sciences and Humanities, University of Wollongong, Wollongong, NSW, Australia \\ ${ }^{2}$ School of Public Health, Faculty of Health Sciences, Curtin University, Perth, WA, Australia \\ ${ }^{3}$ Department of Medical Services, Ministry of Health, Royal Government of Bhutan, Thimphu, Bhutan \\ ${ }^{4}$ Bumthang General Hospital, Ministry of Health, Royal Government of Bhutan, Bumthang, Bhutan
}

Background: Sedentary behavior is associated with several adverse health outcomes. Data on factors that influence sedentary behavior are lacking in Bhutan. This study examined factors associated with increased sedentary behavior in Bhutan, with a focus on exploring sex differences.

Methods: Data of 2,796 adults from the nationally representative 2014 Bhutan STEP-wise surveillance (STEPS) survey were analyzed. Factors associated with sedentary behavior were identified using backward elimination multiple logistic regression analysis, disaggregated by sex. The analysis accounted for the complex survey design used in the primary survey.

Results: The overall prevalence of sedentary behavior was $8.2 \%$, with a higher proportion among women than men ( $10.3 \%$ vs. $4.9 \%$ ). In the full sample, female sex, being single, high education and income, urban residence, inadequate physical activity, and high blood sugar were associated with increased odds of sedentary behavior. Among females, those who had high education and income, were single, physically less active, and urban residents were more likely to be sedentary. Self-employment was related to reduced odds of sedentary behavior among women and in the overall sample population. In males, being single, higher education level, and urban residence were associated with sedentariness.

Conclusion: The findings suggest that interventions targeting females, especially those who are physically less active and from higher socioeconomic groups, urban residents, and those with hyperglycemia can potentially help reduce sedentary behavior and avert the associated detrimental impacts.

Keywords: Sedentary Behavior; Risk Factors; Sitting Position; Bhutan

Received: March 27, 2020, Revised: May 19, 2020, Accepted: May 27, 2020

*Corresponding Author: Tashi Dendup https://orcid.org/0000-0002-6754-6302

Tel: +61-2-42214990, Fax: +61-2-42215257, E-mail: taseedee@gmail.com 


\section{INTRODUCTION}

Sedentary behavior is recognized as an important modifiable health risk behavior distinct from physical inactivity. ${ }^{1)}$ Activities conducted in the sitting position that involve low metabolic energy expenditure ( $\leq 1.5$ metabolic equivalents task) such as watching TV and desk work, computer activities, and sitting during commuting constitute sedentary behavior. ${ }^{2,3)}$ Sedentary behavior can adversely impact physical and mental health and mortality independent of the physical activity level. ${ }^{2,45}$ However, implementing breaks during sedentary activity, such as a short period of standing, and physical activity between prolonged periods of sitting, can potentially benefit physical and metabolic health irrespective of physical activity level and sitting time. ${ }^{6,7)}$ Specific interventions separate from increasing physical activity may be needed to reduce sedentary behavior, given the possible distinct influence of sedentary behavior on overall health. ${ }^{3)}$

Understanding the factors that influence sedentary behavior is crucial to identify the at-risk population and target interventions to reduce sedentary behavior. Current literature shows that age, ${ }^{2,8-10)} \operatorname{sex}^{2,11)}$ marital status, ${ }^{11)}$ education, ${ }^{2,8,11)}$ employment status, ${ }^{2,9,11-13)}$ income, ${ }^{2,8,11,12)}$ ethnicity, ${ }^{9)}$ personal beliefs, ${ }^{2)}$ and area of residence ${ }^{8,13)}$ are sociodemographic correlates of sedentary behavior. Health and behavioral factors, such as obesity, ${ }^{2,8,11,13)}$ insufficient physical activity, ${ }^{2,8,11,13)}$ alcohol use, ${ }^{8)}$ and smoking status, ${ }^{2)}$ were also found to be associated with sedentary behavior. Additionally, individuals with poor physical health, increased prescribed medications, ${ }^{9,13)}$ depressive symptoms, low quality of life, ${ }^{2,14)}$ disability, and chronic conditions ${ }^{9,11,13)}$ have higher odds of sedentary behavior. Environmental factors such as traffic, pollution, safety, lack of green spaces, and lessened access to sidewalks and recreational facilities are also thought to promote sedentary behavior and prevent physical activity. ${ }^{11,15-17)}$

The 2014 Bhutan National Survey for Noncommunicable Disease Risk Factors and Mental Health (referred to as the Bhutan STEPS Survey in the present study) showed that the mean sitting time per day among adults was 148.0 minutes. ${ }^{18)}$ A similar survey of the capital city in 2007 reported a mean time spent in sedentary behavior of 174.2 minutes per day, with higher time among older adults. ${ }^{19)}$ Both surveys reported significantly increased mean sitting time among women compared to men, which may indicate an existing within-population variation in sex. A more recent survey among school-going children found that around $30 \%$ spent $\geq 3$ hours per day engaged in sedentary activities, and only $23.5 \%$ were physically active. ${ }^{20)}$ To date, no studies have explored the factors that influence sedentary behavior in Bhutan. Review studies also found that data on the correlates of sedentary behavior in low- and middle-income countries are limited. ${ }^{11,12)}$ We aimed to bridge this gap in the body of knowledge by examining the factors associated with sedentary behavior among adults using data from the 2014 Bhutan STEPS Survey, with an emphasis on examining sex differences.

\section{METHODS}

\section{Study Data and Sample}

This study used nationally representative, cross-sectional data from the most recent Bhutan STEPS Survey (conducted in 2014), that aplied the World Health Organization STEP-wise surveillance (STEPS) approach ${ }^{18)}$ The survey included men and women between the ages of 18-69 years and provided information on noncommunicable disease prevalence and modifiable risk factors. A multi-stage cluster sampling approach, with distinct rural and urban areas as the primary sampling units, was employed to select the participants in the survey. From these identified clusters, one eligible participant per household was recruited. The data were collected electronically using personal digital assistants between April and June 2014, and the survey achieved a response rate of approximately $97 \%$. The findings and details of the methods of the 2014 Bhutan STEPS Survey have been previously published. ${ }^{18)}$ After excluding 26 individual responses with incomplete information on time spent sitting or reclining, the final cohort for the present study included data from 2,796 participants.

\section{Study Variables}

\section{1) Dependent variable}

Self-reported responses to the question "How much time do you usually spend sitting or reclining on a typical day?" measured in hours and minutes per day were used to extract information on sitting time. Except for time spent sleeping, this question collected data on the total time spent sitting or reclining at work, at home, getting to and from places, or with friends, including time spent sitting at a desk, sitting with friends, traveling on the bus, reading, playing cards, or watching television. Since a high level of sitting is found to adversely impact health, a cut-off of sitting for $\geq 6$ hours per day was used to define sedentary behavior or high sitting time, according to previous studies. ${ }^{9,17)}$

\section{2) Independent variables}

Age (>50, 41-50, 31-40, 18-30 years), sex (male, female), and marital status (single, married/cohabitating, previously married) were the demographic factors used for grouping. Socioeconomic factors used for grouping included education (no education, primary, or secondary), employment status (employed, self-employed, unemployed), household income (divided into tertiles), and place of residence (rural or urban). Health and behavioral factors used for grouping included smoking status (yes or no), alcohol use (yes or no), physical activity (yes or no), high blood pressure (yes or no), high blood sugar (yes or no), body mass index (BMI; normal, underweight, overweight/obese), and suicidal ideation as a proxy for overall mental health (yes or no).

Physical and biochemical measures were used to assess BMI, blood pressure, and blood sugar levels. In line with the Bhutan STEPS Survey report, ${ }^{18)}$ a cut-off of $\geq 140 \mathrm{~mm} \mathrm{Hg}$ for systolic blood pressure and $\geq 90$ $\mathrm{mm} \mathrm{Hg}$ for diastolic blood pressure was used to define high blood pressure. A blood sugar level of $>110 \mathrm{mg} / \mathrm{dL}$ was used to identify those 


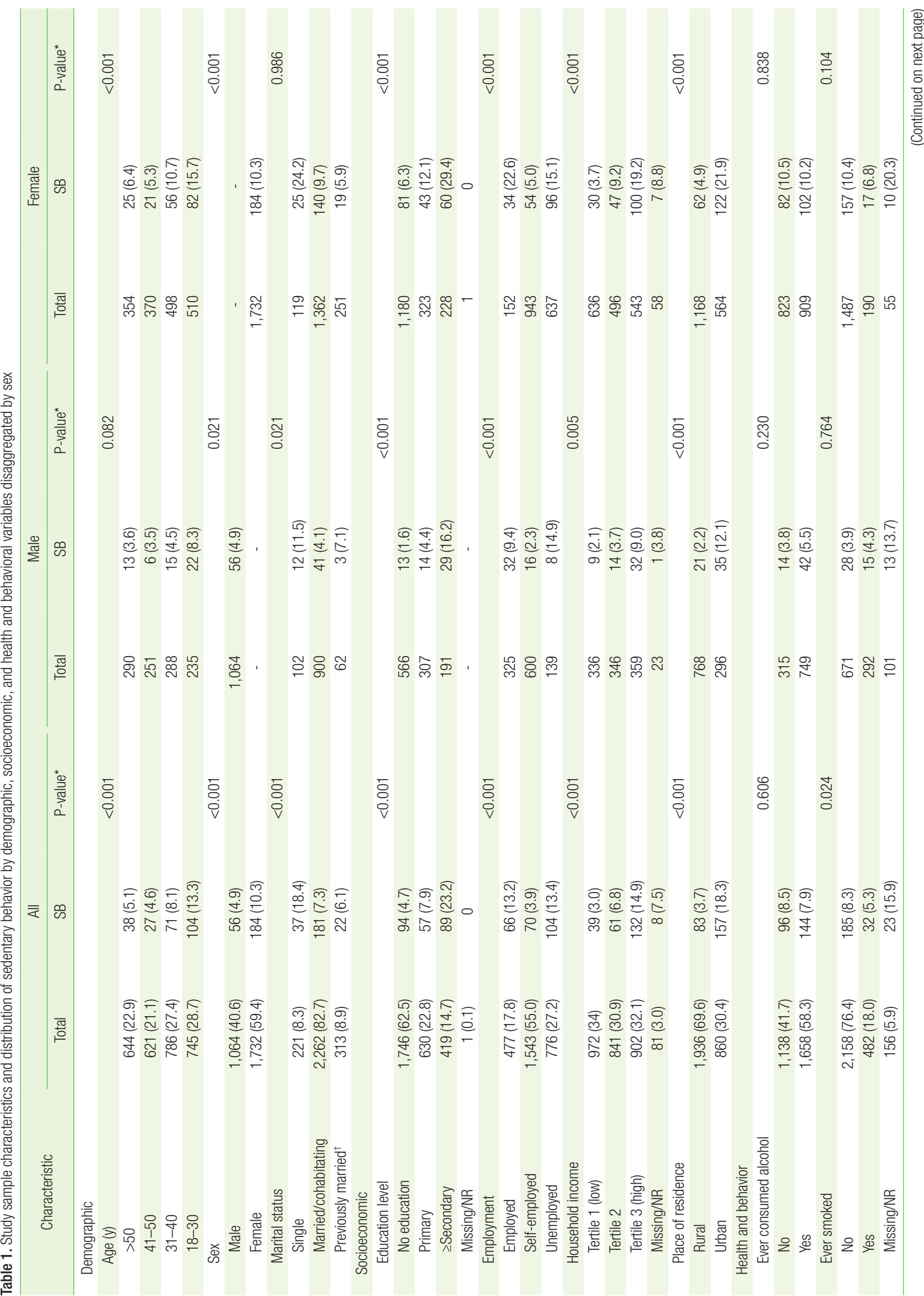




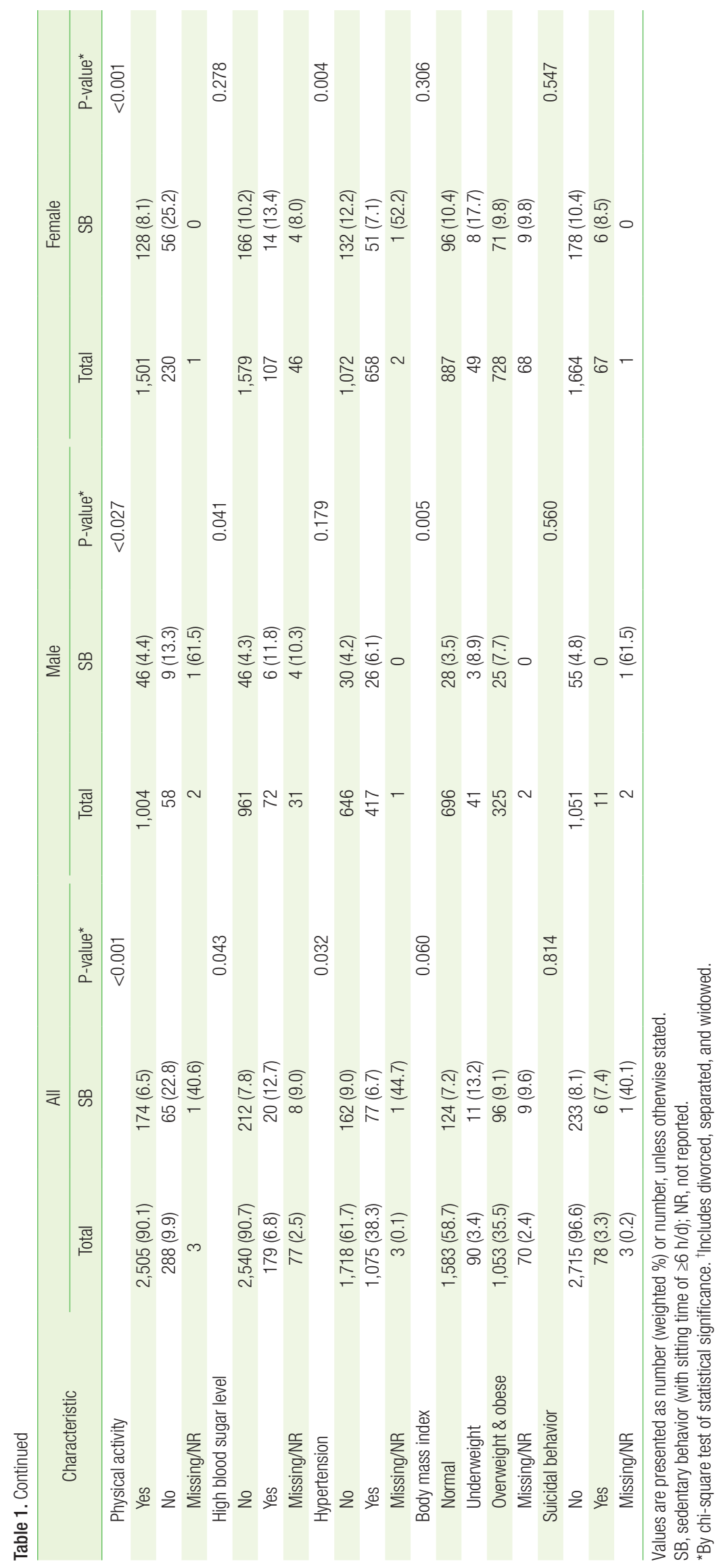


with high blood sugar levels, and a BMI of $\geq 25 \mathrm{~kg} / \mathrm{m}^{2}$ was used to identify those who were overweight or obese. Self-reported "yes" or "no" responses were used to determine the physical activity variable that assessed participation in moderate- and vigorous-intensity work-related activity, sports, fitness or recreational activities. Yes or no responses to the questions "Have you ever consumed any alcohol such as beer, wine, hard drinks, or local drinks (ara, changkoe, or bangchang)?", and "In the past, did you ever smoke any tobacco products?" were used to assess alcohol use and smoking status, respectively.

\section{Data Analysis}

The weighted prevalence of sedentary behavior (defined as a sitting time of $\geq 6$ hours per day) was calculated. The chi-square test was per- formed to assess the differences in sedentary behavior prevalence between the groups of independent variables. Bivariate and multivariable logistic regression analyses were performed. Unadjusted and adjusted odds ratios (AORs) of sedentary behavior and their 95\% confidence intervals (CIs) were reported. A P-value of $<0.05$ was used to determine statistical significance of association in the final model that was built using a backward elimination regression approach. The analyses were carried out separately for the overall sample, and males and females samples. Multicollinearity among the independent variables was assessed using the variance inflation factor (VIF). All data analyses were conducted using STATA ver. 14.0 (Stata Corp., College Station, TX, USA). To account for the complex survey design, stratification, clustering, and sampling weight of the survey, the STATA survey

Table 2. Unadjusted association between the demographic, socioeconomic, and health and behavioral factors and sedentary behavior disaggregated by sex

\begin{tabular}{|c|c|c|c|}
\hline Variable & All & Male & Female \\
\hline \multicolumn{4}{|l|}{ Demographic } \\
\hline \multicolumn{4}{|l|}{ Age (ref: $>50$ y) } \\
\hline $41-50$ & $0.88(0.48-1.63)$ & $0.97(0.28-3.33)$ & $0.82(0.43-1.55)$ \\
\hline $31-40$ & $1.63(0.91-2.92)$ & $1.25(0.55-2.84)$ & $1.74(0.88-3.47)$ \\
\hline $18-30$ & $2.84(1.67-4.84)$ & $2.41(1.05-5.56)$ & $2.73(1.47-5.04)$ \\
\hline \multicolumn{4}{|l|}{ Sex (ref: male) } \\
\hline Female & $2.21(1.57-3.13)$ & - & - \\
\hline \multicolumn{4}{|c|}{ Marital status (ref: married/coupling) } \\
\hline Single & $2.86(1.62-5.07)$ & $3.03(1.50-6.13)$ & $2.98(1.53-5.81)$ \\
\hline Previously married* & $0.83(0.44-1.56)$ & $1.78(0.39-8.17)$ & $0.59(0.30-1.15)$ \\
\hline \multicolumn{4}{|l|}{ Socioeconomic } \\
\hline \multicolumn{4}{|c|}{ Education level (ref: no education) } \\
\hline$\geq$ Primary & $3.29(1.94-5.59)$ & $5.72(2.38-13.74)$ & $3.61(2.19-5.98)$ \\
\hline \multicolumn{4}{|l|}{ Employment (ref: unemployed) } \\
\hline Employed & $0.98(0.57-1.72)$ & $1.67(0.64-4.26)$ & $1.64(0.80-3.36)$ \\
\hline Self-employed & $0.26(0.17-0.41)$ & $0.38(0.13-1.07)$ & $0.30(0.19-0.47)$ \\
\hline \multicolumn{4}{|c|}{ Household income (ref: tertile 1 [low]) } \\
\hline Tertile 2 & $2.33(1.32-4.10)$ & $1.86(0.64-5.37)$ & $2.66(1.31-5.40)$ \\
\hline Tertile 3 (high) & $5.58(3.40-9.13)$ & $4.69(1.52-14.49)$ & $6.28(3.70-10.67)$ \\
\hline \multicolumn{4}{|l|}{ Place of residence (ref: rural) } \\
\hline Urban & $5.76(2.98-11.14)$ & $6.19(2.57-14.94)$ & $5.50(2.85-10.62)$ \\
\hline \multicolumn{4}{|l|}{ Health and behavior } \\
\hline \multicolumn{4}{|c|}{ Ever consumed alcohol (ref: no) } \\
\hline Yes & $0.92(0.66-1.28)$ & $1.48(0.78-2.80)$ & $0.96(0.65-1.42)$ \\
\hline \multicolumn{4}{|l|}{ Ever smoked (ref: no) } \\
\hline Yes & $0.62(0.40-0.94)$ & $1.10(0.59-2.07)$ & $0.63(0.36-1.10)$ \\
\hline \multicolumn{4}{|l|}{ Physical activity (ref: yes) } \\
\hline No & $4.26(2.74-6.62)$ & $3.36(1.08-10.45)$ & $3.84(2.24-6.57)$ \\
\hline \multicolumn{4}{|l|}{ High blood sugar (ref: no) } \\
\hline Yes & $1.73(1.01-2.94)$ & $2.99(0.99-8.98)^{\dagger}$ & $1.37(0.78-2.40)$ \\
\hline \multicolumn{4}{|l|}{ Hypertension (ref: no) } \\
\hline Yes & $0.72(0.54-0.97)$ & $1.46(0.84-2.53)$ & $0.55(0.37-0.83)$ \\
\hline \multicolumn{4}{|l|}{ Body mass index (ref: normal) } \\
\hline Underweight & $1.96(1.02-3.77)$ & $2.71(0.96-7.62)$ & $1.85(0.77-4.47)$ \\
\hline Overweight \& obese & $1.29(0.94-1.76)$ & $2.32(1.38-3.92)$ & $0.94(0.64-1.38)$ \\
\hline \multicolumn{4}{|l|}{ Suicidal behavior (ref: no) } \\
\hline Yes & $0.91(0.43-1.93)$ & - & $0.79(0.37-1.69)$ \\
\hline
\end{tabular}

Values are presented as crude odds ratio (95\% confidence interval). The bold type is considered statistically significant at the $5 \%$ level. Crude/unadjusted odds ratio obtained from simple logistic regression model.

Ref, reference.

${ }^{*}$ Includes divorced, separated, and widowed. ${ }^{\dagger}$ Marginally significant $\mathrm{P}=0.05$. 
(svy) macro was used in the analysis.

\section{RESULTS}

The mean age of the participants was 40.1 years. The overall weighted prevalence of sedentary behavior was $8 \%$. The characteristics of the study sample and the distribution of sedentary behavior are presented in Table 1 for the overall, female, and male sample populations. Overall, the prevalence of sedentary behavior was higher among females $(10.3 \%)$, those who were $\leq 30$ years of age (13.3\%), single individuals (18.4\%), individuals with a secondary level of education or higher (23.2\%), employed (13.2\%) or unemployed (13.4\%) individuals, high income earners (14.9\%), and urban residents (18.3\%). Those who were physically less active (22.8\%), non-smokers (8.3\%), underweight (13.2\%), overweight/obese (9.1\%), had high blood sugar levels (12.7\%), or had normal blood pressure (9\%) also reported higher sedentary behavior. For individual female and male sample groups, similar consistent prevalence and distribution of sedentary behavior were observed for almost all factors.

In the full sample, bivariate analysis showed that females and those who were young, single, had a primary level of education or higher, had medium or high income, and resided in urban areas were more likely to be sedentary (Table 2). The odds of sedentary behavior were also greater among those who were physically less active, underweight, and had high blood sugar, while those who smoked, were selfemployed, and were hypertensive had lower odds. In the male and female subsamples, the associations retained a similar direction for most independent variables. However, in both females and males, smoking and being underweight became non-significant, and being hypertensive was associated in females, while being overweight or obese was assosciated only in males.

For the overall sample, multivariable analysis showed that two demographic factors (sex and marital status), four socioeconomic factors (education, employment, household income, and place of residence), and two health and behavioral factors (physical activity and high blood sugar) were significantly associated with sedentary behavior (Table 3). Specifically, being female (AOR, 2.50; 95\% CI, 1.75-3.58) and single (AOR, 2.22; 95\% CI, 1.27-3.89) were associated with increased odds of sedentary behavior compared to their counterparts. The odds of increased sedentary behavior were also higher among urban residents (AOR, 2.66; 95\% CI, 1.29-5.48), those with a primary level of education or higher (AOR, 1.81; 95\% CI, 1.18-2.79), and those from highincome households (AOR, 2.05; 95\% CI,1.14-3.64). Compared to those who were unemployed, those who were self-employed (AOR, 0.55; 95\% CI, 0.35-0.85) had reduced odds of sedentary behavior. The likelihood of sedentary behavior was 2.25 (95\% CI, 1.28-3.96) and 1.97 (95\%

Table 3. Adjusted odds ratio of the factors associated with sedentary behavior disaggregated by sex

\begin{tabular}{|c|c|c|c|}
\hline Variable & $A l^{*}$ & $\mathrm{Male}^{\dagger}$ & Female $^{\ddagger}$ \\
\hline \multicolumn{4}{|l|}{ Demographic } \\
\hline \multicolumn{4}{|l|}{ Sex (ref: male) } \\
\hline Female & $2.50(1.75-3.58)$ & & \\
\hline \multicolumn{4}{|c|}{ Marital status (ref: married/coupling) } \\
\hline Single & $2.22(1.27-3.89)$ & 2.40 (1.18-4.88) & $2.11(1.09-4.09)$ \\
\hline Previously married ${ }^{\S}$ & $1.13(0.58-2.23)$ & $2.13(0.50-9.14)$ & $1.03(0.50-2.10)$ \\
\hline \multicolumn{4}{|l|}{ Socioeconomic } \\
\hline \multicolumn{4}{|c|}{ Education level (ref: no education) } \\
\hline$\geq$ Primary & $1.81(1.18-2.79)$ & 2.35 (1.48-7.58) & $1.57(1.01-1.43)$ \\
\hline \multicolumn{4}{|l|}{ Employment (ref: unemployed) } \\
\hline Employed & $0.97(0.54-1.73)$ & & $0.99(0.46-2.12)$ \\
\hline Self-employed & $0.55(0.35-0.85)$ & & $0.52(0.32-0.85)$ \\
\hline \multicolumn{4}{|c|}{ Household income (ref: tertile 1 [low]) } \\
\hline Tertile 2 & $1.67(0.91-3.03)$ & & $1.68(0.82-3.44)$ \\
\hline Tertile 3 (high) & $2.04(1.14-3.64)$ & & $2.26(1.13-4.53)$ \\
\hline \multicolumn{4}{|l|}{ Place of residence (ref: rural) } \\
\hline Urban & $2.66(1.29-5.48)$ & 4.49 (1.87-10.77) & $2.60(1.18-5.73)$ \\
\hline \multicolumn{4}{|l|}{ Health and behavior } \\
\hline \multicolumn{4}{|l|}{ Physical activity (ref: yes) } \\
\hline No & $2.25(1.28-3.96)$ & & $2.27(1.22-4.25)$ \\
\hline \multicolumn{4}{|l|}{ High blood sugar (ref: no) } \\
\hline Yes & $1.97(1.13-3.45)$ & & \\
\hline
\end{tabular}

Values are presented as adjusted odds ratio ${ }^{\ddagger}(95 \%$ confidence interval). The bold type is considered statistically significant at the $5 \%$ level. Empty cells indicate these variables were not statistically significant in the final multivariable model obtained using backward elimination in each sample group. Adjusted odds ratio obtained from multivariable logistic regression model built using backward elimination.

Ref, reference; BMl, body mass index.

*Model adjusted for age, sex, marital status, education, employment, household income, place of residence, smoking, physical activity, blood sugar, hypertension, and BMl. ${ }^{\dagger}$ Model adjusted for age, marital status, education, employment, household income, place of residence, alcohol, physical activity, blood sugar, and BMl. ${ }^{\ddagger}$ Model adjusted for

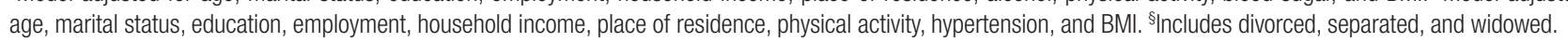


CI, 1.13-3.45) times higher among those who were physically less active and who had high blood sugar, respectively.

For the female subsample, being single, having primary level of education or higher, residing in urban areas, and being physically less active were associated with a higher likelihood of having sedentary behavior. By contrast, self-employed females had reduced odds of sedentary behavior compared to those who were unemployed. In the male subsample, similar to females, those who were single, had a primary level of education or higher, and resided in urban areas had a higher likelihood of being sedentary, and the association for these variables appeared more pronounced in males. However, employment, household income, and physical activity were not significant factors influencing sedentary behavior among men. The VIFs were less than 10, indicating that multicollinearity was not an issue in the regression analysis.

\section{DISCUSSION}

To our knowledge, this is the first study from Bhutan that examined factors associated with sedentary behavior with comparison of sex differences. The overall prevalence of sedentary behavior was $8.2 \%$. With a two-fold significantly higher prevalence among females, roughly one in 10 Bhutanese women were sedentary. A study that examined nationally representative data from six low- and middle-income countries also reported an overall sedentary behavior prevalence of $8.3 \%$, although the prevalence varied from $4.6 \%$ in South Africa to as high as 17.7\% in Russia. ${ }^{13)}$ Analysis of the total sample population demonstrated that being female, being single, high education level, high income, urban residence, high blood sugar levels, and inadequate physical activity were correlated with sedentary behavior. For the subsamples separated by sex, the common factors of sedentary behavior were being single, having primary education level or higher, and urban residence. Being self-employed was associated with reduced odds of sedentary behavior among females as well as the overall sample population. Particularly for females, the odds of sedentary behavior were higher among those who were physically less active and had a high income.

Consistent with the data in the literature, ${ }^{11,21,22)}$ our study found that females were more likely to engage in sedentary behavior than males. Bhutanese women are more likely to have a higher body mass index, are physically less active ${ }^{18)}$ and experience more unpleasant feelings or emotions such as anxiety, sadness, and other signs of mental illhealth. ${ }^{23)}$ Inadequate physical activity, high body weight, and poor mental health are correlated with sedentary behavior. ${ }^{2,8,13,14)}$ Moreover, our results also showed that less physically active females were more likely to be sedentary. Similar to the findings of previous studies, ${ }^{9,21)}$ our findings suggest that being married was associated with reduced odds of high sitting time among both men and women. Married individuals may have children, and the increased associated responsibility may necessitate more physical movement. Studies have also shown that having children is associated with a lower sitting time. ${ }^{2,11)}$ Single individuals may spend more time using computers and watching TV than those married; ${ }^{22)}$ thus, they may be more sedentary.

Although young individuals tended to be more sedentary, this association was not significant in the multivariate analysis. Older adults ( $\geq 60$ years of age) are shown to have higher levels of sedentary behavior that increases with age, with more elderly males spending more time engaged in sedentary activities than females. ${ }^{10,11,22)}$ Only individuals aged 18-69 years were recruited for the 2014 Bhutan STEPS Survey; therefore, our data on older individuals is somewhat limited. The available data suggest that age is positively associated with TV viewing and negatively associated with computer use, while the findings are mixed for generalized screen time and sitting. ${ }^{2)}$ The lack of information on domain-specific sedentary activities and limited age range of individuals assessed might explain this discrepancy.

Our findings showed that education level and household income were directly associated with sedentary behavior. Our results replicate the data in the literature that show a positive association between socioeconomic status and sedentary behavior. ${ }^{11,12,17,21)}$ Greater use of motor vehicles and technology and sedentary occupations may explain this association. Easy access to electronic gadgets, such as computers and notepads, can lead to increased screen time. Moreover, those with higher education levels may be more likely to be employed in professions that involve sitting while working. ${ }^{11)}$ Nevertheless, higher education level and employment are also associated with lower leisure-time sedentary behavior. ${ }^{12)}$ This sitting duration could have been captured in the total sitting time in our study.

Females from wealthier households had higher odds of sedentary behavior, while self-employed females had lower odds. Owing to financial security, women from wealthy households may be less likely to undertake paid jobs or perform daily household chores, increasing the probability of being sedentary. Self-employment is associated with better physical and mental health. ${ }^{24)}$ Psychological benefits from greater autonomy and the consequent physical health gains, and flexible working hours that may enable healthy behaviors such as physical activity, may provide some explanation. ${ }^{24)}$ Self-employed females may have better control over the way they work and may use skills that enable them to engage in healthy behaviors. A previous study showed that self-employed females reported a higher level of well-being, which may be attributable to better autonomy related to being selfemployed. ${ }^{25)}$ However, being unemployed was found to be associated with sedentary behavior. ${ }^{13)}$ Those unemployed may have more time at their disposition for leisure activities, such as watching $\mathrm{TV}^{26)}$ They may also opt for cheaper recreational activities such as TV, and time spent watching TV can reduce the time for higher intensity physical activity. ${ }^{2)}$ Moreover, unemployed individuals may have other chronic conditions that may prohibit physical movement. ${ }^{13)}$ In the Asian context, low priority for physical activity, upholding cultural traditions, lack of support, and perceived physical appearance may affect physical activity among women. ${ }^{27)}$ The unemployed category in our study included those who were retired, unable to work, and homemakers. In Bhutan, homemakers are usually female, and our data suggest that females are more sed- 
entary.

In agreement with the data in the literature, ${ }^{12,13}$ our study found that those living in urban areas were more likely to be sedentary than their rural counterparts. Rural employment usually consists of agricultural jobs requiring physical movement, whereas the higher use of technology in urban settings may facilitate sedentary behavior. In Bhutan, rural residents largely depend on farming for their livelihood. Furthermore, urban environmental features, such as high traffic and crime, may promote prolonged sitting. ${ }^{11,15)}$ Presently, urban Bhutan is experiencing increases in traffic, air pollution, and crime partly driven by an increase in rural-urban migration. ${ }^{28,29}$ These environmental attributes may also promote the use of cars and affect mental health, which, in turn, can influence sedentary behavior.

Inadequate physical activity and high blood sugar levels were correlated with sedentary behavior. This aligns with data in the literature. ${ }^{9,11,13,17)}$ Those with chronic conditions might be physically less active and may have a greater number of comorbid conditions associated with sedentary behavior, possibly due to mobility restrictions, discomfort, or mental health problems. ${ }^{13)}$ However, sedentary behavior may also predict blood sugar and physical activity levels. The crosssectional study design prevents the assessment of the directionality of the association. The findings regarding inadequate physical activity may be more relevant to the development of targeted interventions, given that high physical activity may potentially attenuate the detrimental effects of sedentary behavior. ${ }^{30)}$ The association for physical activity was robust only among females, which supports data indicating that Bhutanese women are likely to be less physically active than men. ${ }^{18)}$ We found no strong evidence for an association between body weight and sedentariness, which supports the findings of a previous systematic review ${ }^{4)}$ and study. ${ }^{17)}$ However, specific measures of sitting time, such as TV viewing, were found to be associated with being overweight ${ }^{31}$ implying that the association may depend on domain-specific sedentary behavior.

\section{Strengths and Limitations}

The strengths of our study include the use of the latest nationally representative data with a high response rate (approximately 97\%) obtained from the Bhutan STEPS Survey. With the availability of this large sample size, we were able to perform additional analyses for sexdisaggregated subsamples. Moreover, we accounted for the complex survey design, clustering, and sampling weights in our analyses. The findings reflect lifestyle-related public health concerns in modern Bhutan and can be widely applied to the general population.

This study had some limitations. First, given its high prevalence and passive nature, self-reported sedentary behavior is susceptible to recall and social desirability bias. Self-reported measures were found to be much lower than objectively measured sedentary behavior. ${ }^{10)}$ This could have attenuated the associations to some extent. Owing to the lack of information in the Bhutan STEPS Survey data, we could not explore the influence of some physical and mental health-related, psychosocial, and environmental factors that are shown to be important sedentary behavior correlates. ${ }^{11,12,14,15)}$ Future studies may consider collecting information on specific types of sedentary behavior and examine social and environmental correlates that may enable targeted interventions, given that these factors are modifiable. Finally, the crosssectional design of the study prohibits inferring the directionality of the associations found.

\section{Conclusions}

Our findings showed that $8.2 \%$ of Bhutanese adults were sedentary, and that the prevalence of sedentary behavior was higher among women. Overall, being female, being single, having higher education and income levels, urban residence, high blood sugar levels, and inadequate physical activity were associated with higher odds of sedentary behavior. These findings suggest that socioeconomic and behavioral factors may be more important in influencing sedentary behavior among females. Like many developing countries, chronic conditions and diseases such as obesity, hypertension, type 2 diabetes, and cardiovascular diseases are on the rise in Bhutan. ${ }^{18)}$ Given the impact of physical inactivity and prolonged sitting on the risk of developing these conditions, the findings may help lower the burden of these conditions by informing policy development aimed at reducing sedentariness and increasing physical activity.

Policy interventions targeting higher socioeconomic groups, urban residents, and those with high blood sugar levels may help reduce sedentary behavior. In addition, interventions focusing especially on females who are physically less active and/or unemployed could be cost-effective. Policies aimed to create environments which facilitate transportation, occupational and recreational opportunities that reduce prolonged sitting may help avoid the adverse effects of sedentary behavior.

\section{CONFLICT OF INTEREST}

No potential conflict of interest relevant to this article was reported.

\section{ACKNOWLEDGMENTS}

We would like to thank the Ministry of Health, Royal Government of Bhutan for granting access to the 2014 Bhutan STEPS Survey dataset. We also thank all the participants of the Bhutan STEPS Survey.

\section{ORCID}

Tashi Dendup: https://orcid.org/0000-0002-6754-6302

Yun Zhao: https://orcid.org/0000-0001-8445-8630

I Gusti Ngurah Edi Putra: https://orcid.org/0000-0002-1014-6949

Tandin Dorji: https://orcid.org/0000-0003-2698-9067

Chador Tenzin: https://orcid.org/0000-0003-4993-4624 


\section{REFERENCES}

1. Owen N, Healy GN, Matthews CE, Dunstan DW. Too much sitting: the population health science of sedentary behavior. Exerc Sport Sci Rev 2010;38:105-13.

2. Rhodes RE, Mark RS, Temmel CP. Adult sedentary behavior: a systematic review. Am J Prev Med 2012;42:e3-28.

3. Gonzalez K, Fuentes J, Marquez JL. Physical inactivity, sedentary behavior and chronic diseases. Korean J Fam Med 2017;38:111-5.

4. Thorp AA, Owen N, Neuhaus M, Dunstan DW. Sedentary behaviors and subsequent health outcomes in adults a systematic review of longitudinal studies, 1996-2011. Am J Prev Med 2011;41:207-15.

5. Sloan RA, Sawada SS, Girdano D, Liu YT, Biddle SJ, Blair SN. Associations of sedentary behavior and physical activity with psychological distress: a cross-sectional study from Singapore. BMC Public Health 2013;13:885.

6. Healy GN, Dunstan DW, Salmon J, Cerin E, Shaw JE, Zimmet PZ, et al. Breaks in sedentary time: beneficial associations with metabolic risk. Diabetes Care 2008;31:661-6.

7. Garber CE, Blissmer B, Deschenes MR, Franklin BA, Lamonte MJ, Lee IM, et al. American College of Sports Medicine position stand: quantity and quality of exercise for developing and maintaining cardiorespiratory, musculoskeletal, and neuromotor fitness in apparently healthy adults: guidance for prescribing exercise. Med Sci Sports Exerc 2011; 43:1334-59.

8. Dias PJ, Domingos IP, Ferreira MG, Muraro AP, Sichieri R, GoncalvesSilva RM. Prevalence and factors associated with sedentary behavior in adolescents. Rev Saude Publica 2014;48:266-74.

9. Jamil AT, Rosli NM, Ismail A, Idris IB, Omar A. Prevalence and risk factors for sedentary behavior among Malaysian adults. Malays J Public Health Med 2016;16:147-55.

10. Harvey JA, Chastin SF, Skelton DA. How sedentary are older people?: a systematic review of the amount of sedentary behavior. J Aging Phys Act 2015;23:471-87.

11. O’Donoghue G, Perchoux C, Mensah K, Lakerveld J, van der Ploeg H, Bernaards C, et al. A systematic review of correlates of sedentary behaviour in adults aged 18-65 years: a socio-ecological approach. BMC Public Health 2016;16:163.

12. Prince SA, Reed JL, McFetridge C, Tremblay MS, Reid RD. Correlates of sedentary behaviour in adults: a systematic review. Obes Rev 2017;18:915-35.

13. Koyanagi A, Stubbs B, Vancampfort D. Correlates of sedentary behavior in the general population: a cross-sectional study using nationally representative data from six low- and middle-income countries. PLoS One 2018;13:e0202222.

14. Hoare E, Milton K, Foster C, Allender S. The associations between sedentary behaviour and mental health among adolescents: a systematic review. Int J Behav Nutr Phys Act 2016;13:108.

15. Oyeyemi AL, Kolo SM, Rufai AA, Oyeyemi AY, Omotara BA, Sallis JF. Associations of neighborhood walkability with sedentary time in Nige- rian older adults. Int J Environ Res Public Health 2019;16:1879.

16. Lin CY, Park JH, Hsueh MC, Lai TF, Liao Y. Are area-level crimes associated with older adults' physical activity and sedentary behavior? Sustainability 2019;11:2454.

17. Wallmann-Sperlich B, Bucksch J, Hansen S, Schantz P, Froboese I. Sitting time in Germany: an analysis of socio-demographic and environmental correlates. BMC Public Health 2013;13:196.

18. World Health Organization. National survey for noncommunicable disease risk factors and mental health using WHO STEPS approach in Bhutan-2014. Geneva: World Health Organization; 2015.

19. Cowan M, Dorji G, Pelzom D. Report on 2007 STEPS Survey for risk factors and prevalence of noncommunicable diseases in Thimphu. Thimphu (Bhutan): Ministry of Health; 2009.

20. Ministry of Health. Report on Bhutan Global School-Based Student Health Survey 2016. Thimphu (Bhutan): Ministry of Health; 2017.

21. Uijtdewilligen L, Yin JD, van der Ploeg HP, Muller-Riemenschneider F. Correlates of occupational, leisure and total sitting time in working adults: results from the Singapore multi-ethnic cohort. Int J Behav Nutr Phys Act 2017;14:169.

22. Shields M, Tremblay MS. Screen time among Canadian adults: a profile. Health Rep 2008;19:31-43.

23. Centre for Bhutan Studies \& GNH Research. A compass towards a just and harmonious society: 2015 GNH survey report. Thimphu (Bhutan): Centre for Bhutan Studies \& GNH Research, Royal Government of Bhutan; 2016.

24. Nikolova M. Switching to self-employment can be good for your health. J Bus Ventur 2019;34:664-91.

25. Nordenmark M, Vinberg S, Strandh M. Job control and demands, work-life balance and wellbeing among self-employed men and women in Europe. Vulnerable Groups Incl 2012;3:18896.

26. Clark BK, Sugiyama T, Healy GN, Salmon J, Dunstan DW, Shaw JE, et al. Socio-demographic correlates of prolonged television viewing time in Australian men and women: the AusDiab study. J Phys Act Health 2010;7:595-601.

27. Im EO, Ko Y, Hwang H, Chee W, Stuifbergen A, Lee H, et al. Asian American midlife women's attitudes toward physical activity. J Obstet Gynecol Neonatal Nurs 2012;41:650-8.

28. Royal Bhutan Police. Statistical yearbook 2017. Thimphu (Bhutan): Royal Bhutan Police; 2017.

29. Asian Development Bank. Bhutan transport 2040: integrated strategic vision. Mandaluyong (Philippines): Asian Development Bank; 2013.

30. Ekelund U, Steene-Johannessen J, Brown WJ, Fagerland MW, Owen N, Powell KE, et al. Does physical activity attenuate, or even eliminate, the detrimental association of sitting time with mortality?: a harmonised meta-analysis of data from more than 1 million men and women. Lancet 2016;388:1302-10.

31. Williams DM, Raynor HA, Ciccolo JT. A review of TV viewing and its association with health outcomes in adults. Am J Lifestyle Med 2008;2: 250-9. 